\title{
Relay Assisted Cooperative OSTBC Communication with SNR Imbalance and Channel Estimation Errors
}

\author{
Bo Niu \\ Department of Electrical and Computer Engineering, \\ New Jersey Institute of Technology \\ Newark, NJ 07102, USA \\ bo.niu@njit.edu
}

\author{
Mihaela C. Beluri, Zinan Lin, Prabhakar Chitrapu \\ InterDigital \\ King of Prussia, PA 19406, USA \\ \{mihaela.beluri, zinan.lin, prabhakar.chitrapu\}@interdigital.com
}

\begin{abstract}
In this paper, a two-hop relay assisted cooperative Orthogonal Space-Time Block Codes (OSTBC) transmission scheme is considered for the downlink communication of a cellular system, where the base station (BS) and the relay station (RS) cooperate and transmit data to the user equipment (UE) in a distributed fashion. We analyze the impact of the SNR imbalance between the BS-UE and RS-UE links, as well as the imperfect channel estimation at the $\mathrm{UE}$ receiver. The performance is analyzed in the presence of Rayleigh flat fading and our results show that the SNR imbalance does not impact the spatial diversity order. On the other hand, channel estimation errors have a larger impact on the system performance. Simulation results are then provided to confirm the analysis.
\end{abstract}

\section{INTRODUCTION}

Cooperative diversity capitalizes on transmissions from antennas at different nodes in order to provide spatial diversity, thus enhancing the performance of a wireless network [1]. Utilizing distributed Orthogonal Space-Time Block Codes [2] [3] has been proven to be effective in realizing the cooperative diversity [4] [5]. Various schemes have been proposed for this purpose [6]-[9]. Using relay to help the transmission is a popular choice. A virtual antenna array is created with source nodes and relay nodes to implement the cooperative transmission. The relay can be either amplify-and-forward (AF) or decode-and-forward (DF). Detail analysis of this two schemes can be found in [1] [4].

In our recent work [10], we proposed a relay assisted cooperative transmission scheme for the Long Term Evolution (LTE)-Advanced system. It has been shown that this scheme, by employing $\mathrm{RS}$ in the cellular network and working with a two-hop cooperative diversity scheme, significantly improves the system coverage and capacity. In the first hop of the transmission scheme, both the RS and UE receive data from the BS. We assume that the BS has a better channel condition to the RS than to the UE. Thus the RS successfully decodes the data before the UE does. In the second hop, the BS and RS

This work was conducted while the first author was employed as a summer intern with the CTO Office, InterDigital. cooperate and transmit data to the UE. The UE then decodes the received data that is transmitted from both the BS and RS. Details of the relay assisted transmission scheme can be found in [10]. In this paper, we focus on the cooperative transmission and its reception in the second hop.

The performance of the cooperative diversity transmission scheme used in the second hop may be impacted by the impairments associated with a practical receiver implementation as well as by the distributed nature of the cooperation scheme. The impairments analyzed in this paper are: 1) the SNR imbalance between the BS-UE and RS-UE links, and 2) channel estimation errors at the UE receiver. In practical deployment scenarios, the transmit powers of the BS and the RS may be different. Additionally, the propagation loss on the BS-UE link is likely different from the propagation loss on the RS-UE link. The different transmit powers and propagation losses for the two links, in conjunction with imperfect power control, may lead to SNR imbalance between the BS-UE and RS-UE links (with possibly a better SNR for the RS-UE link). This may result in performance degradation as compared to the case of equal SNR per each link. To evaluate the impact of channel estimation errors, an MMSE estimator is used in the literature, and the channel estimation error is assumed to be a zero mean complex Gaussian random variable and independent of the channel [11]. The performance of the OSTBC scheme with imperfect channel estimation has been analyzed for the Alamouti case in [12], but to the best of our knowledge, not for the case of distributed antennas with SNR imbalance and channel estimation errors.

\section{Contributions and Relation to Previous Work}

In this work, we consider realistic scenarios for a relay assisted wireless cellular system and shed light on issues that deserve attention during practical network design. We study the performance of a cooperative OSTBC diversity scheme with the consideration of SNR imbalance and channel estimation errors, which are impairments that are likely to 
occur in practice. The system setup is similar to [10], except that we introduce the OSTBC transmission scheme in the second hop to achieve spatial diversity, and improve the system performance. The previous result of [12] is obtained as a special case of our work, for no SNR imbalance. The detailed contributions reported in this paper can be summarized as follows:

- Derivation of the theoretical probability of error performance (BER vs. SNR) for the cooperative $2 \times 1$ OSTBC (Alamouti) scheme with SNR imbalance between the RS-UE and the BS-UE link.

- Proof that the SNR imbalance between the RS-UE and the BS-UE link does not affect the spatial diversity of the cooperative system.

- Simulation results that show the channel estimation errors, compared to SNR imbalance, have a major effect on the cooperative OSTBC system performance.

The rest of the paper is organized as follows. In the following section, the relay assisted cooperative OSTBC communication system is introduced. We especially focus on the second hop transmission of the scheme where the BS cooperates with the RS and transmits data to the UE. In Section III, we analyze the system performance degradation due to SNR imbalance between the BS-UE and RS-UE links. We derive the theoretical closed-form probability of error expression for the cooperative Alamouti scheme with SNR imbalance, and prove that the SNR imbalance does not affect the spatial diversity of the scheme. After that, we investigate the effect of channel estimation errors on the system performance in Section IV. Simulation results are then provided in Section $\mathrm{V}$ to confirm our analytical results. Concluding remarks are offered in Section VI.

Notation: Throughout the paper, we denote matrices and vectors with bold face type, using capital letters for matrices and lower case letters for vectors. For any matrix $\mathbf{A}$, the superscript ${ }^{*}$ denotes complex conjugate transpose.

\section{SySTEM MODEL}

In this section, the system model for the relay assisted cooperative OSTBC communication system is introduced. We consider the downlink transmission in a cellular system, where a BS transmits data to UEs. We assume that there is also a RS in the system in order to assist the transmission. As we mentioned before we focus on the second hop transmission of [10] when both the BS and RS have a copy of the transmitted signal (for example, Decode-and-Forward transmission is performed in the first hop and the RS has successfully decoded the data transmitted from BS). The antennas at the BS and RS create a virtual multiple antenna transmitter, encode the data with OSTBC and transmit the coded data cooperatively utilizing all their antennas. For the ease of the theoretical analysis in the following sections, we investigate the cooperative Alamouti scheme for our relay assisted communication. We assume the BS and RS each have one transmit antenna and the UE has one receive antenna. The case of cooperative OSTBC communication with $M_{B}, M_{R}$ transmit antennas at the BS and relay, respectively, and $N$ receive antennas at the UE can be easily generalized.

With the system model introduced above, the received signal vector $\mathbf{y}=\left[\begin{array}{ll}y_{t 0} & y_{t 1}\end{array}\right]$ at the UE can be represented as

$$
\mathbf{y}=\sqrt{P_{B}} h_{B} \mathbf{s}_{B}+\sqrt{P_{R}} h_{R} \mathbf{s}_{R}+\mathbf{z},
$$

where $h_{B}$ and $h_{R}$ represent the Rayleigh fading channel gains for the BS-UE and RS-UE links, respectively. Both of them have i.i.d, complex Gaussian distributions with zero mean and unit variance $\sigma_{n}^{2} . \mathbf{s}_{B}$ and $\mathbf{s}_{R}$ are the $1 \times 2$ transmitted signal vectors from the BS and RS, respectively. They form the Alamouti code as

$$
\mathbf{S}=\left[\begin{array}{l}
\mathbf{s}_{B} \\
\mathbf{s}_{R}
\end{array}\right]=\left[\begin{array}{cc}
s_{0} & -s_{1}^{*} \\
s_{1} & s_{0}^{*}
\end{array}\right],
$$

where $s_{0}, s_{1}$ are transmitted symbols drawn from an equuiprobable source. $\mathbf{z}$ is the received noise vector at the UE. Each entry in $\mathbf{z}$ is a complex Gaussian random variable with zero mean and unit variance; $P_{B}$ and $P_{R}$ are the received powers at the UE due to the transmission from the BS and RS, respectively. The total power is $P=P_{B}+P_{R}$.

In a practical system, $P_{B}$ and $P_{R}$ are generally not the same, which leads to the SNR imbalance in a relay assisted cooperative system. The received SNR for the BS-UE link and RS-UE link are available at the UE since it is able to perform channel estimation of the BS-UE and RS-UE links separately based on different reference symbols. The received reference pilot symbols from the BS and RS are then scaled differently according to the transmitted power and the path loss. When considering the SNR imbalance, we define as $r$ the SNR ratio between the BS-UE and RS-UE links. Thus (1) can be rewritten as

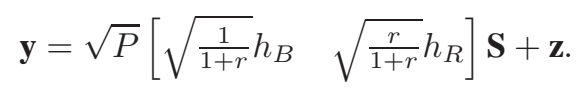

Upon receiving $\mathbf{y}$, the UE performs the revised Alamouti decoding, which considers the SNR imbalance $r$ between RSUE and BS-UE links, by using the estimated channel state information $\hat{h}_{B}$ and $\hat{h}_{R}$. The decoded symbols are

$$
\left[\begin{array}{c}
\tilde{s}_{0} \\
\tilde{s}_{1}
\end{array}\right]=\left[\begin{array}{cc}
\sqrt{\frac{1}{1+r}} \hat{h}_{B}^{*} & \sqrt{\frac{r}{1+r}} \hat{h}_{R} \\
\sqrt{\frac{r}{1+r}} \hat{h}_{R}^{*} & -\sqrt{\frac{1}{1+r}} \hat{h}_{B}
\end{array}\right]\left[\begin{array}{c}
y_{t 0} \\
y_{t 1}^{*}
\end{array}\right] .
$$

In practice, there are errors during the channel estimation process. Following [11], we assume

$$
\hat{h}_{B}=h_{B}+n_{B},
$$

and

$$
\hat{h}_{R}=h_{R}+n_{R},
$$

where $n_{B}$ and $n_{R}$ are the estimation errors, assumed to be independent zero mean complex Gaussian random variables with variance $\beta$.

In the following sections, we investigate the effect of SNR imbalance and channel estimation errors to the performance of the relay assisted cooperative diversity scheme. 


\section{Cooperative Alamouti Diversity SCHEME With SNR IMBALANCE}

In this section, we analyze the impact of the SNR imbalance between the BS-UE and RS-UE links. We assume that there are no channel estimation errors at the UE, i.e., $\hat{h}_{B}=h_{B}$ and $\hat{h}_{R}=h_{R}$.

\section{A. Performance Analysis for Cooperative Alamouti diversity scheme with SNR Imbalance}

Considering the SNR imbalance, the decoded symbol vector (3) can be rewritten as

$$
\begin{aligned}
{\left[\begin{array}{c}
\tilde{s}_{0} \\
\tilde{s}_{1}
\end{array}\right] } & =\sqrt{P}\left[\begin{array}{l}
\left(\frac{1}{1+r}\left|h_{B}\right|^{2}+\frac{r}{1+r}\left|h_{R}\right|^{2}\right) s_{0} \\
\left(\frac{r}{1+r}\left|h_{R}\right|^{2}+\frac{1}{1+r}\left|h_{B}\right|^{2}\right) s_{1}
\end{array}\right] \\
+ & {\left[\begin{array}{l}
\sqrt{\frac{1}{1+r}} h_{B}^{*} z_{0}+\sqrt{\frac{r}{1+r}} h_{R} z_{1}^{*} \\
\sqrt{\frac{r}{1+r}} h_{R}^{*} z_{0}-\sqrt{\frac{1}{1+r}} h_{B} z_{1}^{*}
\end{array}\right] . }
\end{aligned}
$$

It can be readily shown that $\sqrt{\frac{1}{1+r}} h_{B}^{*} z_{0}, \sqrt{\frac{r}{1+r}} h_{R} z_{1}^{*}$ and $\quad \sqrt{\frac{r}{1+r}} h_{R}^{*} z_{0}, \quad \sqrt{\frac{1}{1+r}} h_{B} z_{1}^{*}$ are independent, zero mean Gaussian random variables. Thus $\tilde{s}_{0}$ is the sum of $\left(\frac{1}{1+r}\left|h_{B}\right|^{2}+\frac{r}{1+r}\left|h_{R}\right|^{2}\right) s_{0} \quad$ and $\quad$ an independent, zero mean Gaussian random variable with variance $\left(\frac{1}{1+r}\left|h_{B}\right|^{2}+\frac{r}{1+r}\left|h_{R}\right|^{2}\right)$. Similarly, $\tilde{s}_{1} \quad$ is the sum of $\left(\frac{r}{1+r}\left|h_{R}\right|^{2}+\frac{1}{1+r}\left|h_{B}\right|^{2}\right) s_{1} \quad$ and $\quad$ an independent zero mean Gaussian random variable with variance $\left(\frac{r}{1+r}\left|h_{R}\right|^{2}+\frac{1}{1+r}\left|h_{B}\right|^{2}\right)$. Since $s_{0}$ and $s_{1}$ are drawn from an equiprobable source, we have the following proposition.

Proposition 1 The probability of error performance of the cooperative $2 \times 1$ Alamouti scheme with SNR imbalance $r$ between the $R S-U E$ and the $B S-U E$ links is given by

$$
\begin{aligned}
P e= & \frac{1}{2}\left(1-\frac{1}{\sqrt{1+\frac{2(1+r)}{a^{2} r \gamma}}}\right)\left(1-\frac{1}{\sqrt{1+\frac{2(1+r)}{a^{2} \gamma}}}\right) \\
& \left(1+\frac{1}{\sqrt{1+\frac{2(1+r)}{a^{2} r \gamma}}+\sqrt{1+\frac{2(1+r)}{a^{2} \gamma}}}\right),
\end{aligned}
$$

where $a$ is a constant that depends on the specific modulation mode (e.g. for BPSK, $a=\sqrt{2}$ and for QPSK, $a=1$ ), and $\gamma=P / \sigma_{n}^{2}$.

Proof: See Appendix A.

\section{B. Diversity of Cooperative Alamouti Scheme with SNR Im- balance}

We now look at the spatial diversity of the relay assisted cooperative Alamouti scheme and investigate the effect of SNR imbalance on the diversity order.

Proposition 2 The diversity order of the relay assisted cooperative Alamouti scheme with SNR imbalance between the BS-UE and RS-UE links is 2, which is the same as the regular Alamouti scheme. Thus the SNR imbalance does not affect the diversity order.
Proof: We start from the definition of diversity order in [13],

$$
d=-\lim _{\gamma \rightarrow \infty} \frac{\log P e(\gamma)}{\log \gamma},
$$

and the error of probability in (7), the diversity order of the relay assisted cooperative Alamouti scheme with SNR imbalance can be written as,

$$
\begin{gathered}
d_{s}=-\lim _{\gamma \rightarrow \infty} \frac{\log \left(1-\frac{1}{\sqrt{1+\frac{2(1+r)}{a^{2} r \gamma}}}\right)}{\log \gamma}+\frac{\log \left(1-\frac{1}{\sqrt{1+\frac{2(1+r)}{a^{2} \gamma}}}\right)}{\log \gamma} \\
+\frac{\log \left(1+\frac{1}{\sqrt{1+\frac{2(1+r)}{a^{2} r \gamma}}+\sqrt{1+\frac{2(1+r)}{a^{2} \gamma}}}\right)}{\log \gamma}
\end{gathered}
$$

At high SNR, the Taylor series expansion yields [14]

$$
\sqrt{\frac{B}{A+B}}=1-\frac{A}{2 B}+\mathcal{O}\left(\frac{1}{B^{2}}\right)
$$

Introducing (10) in (9), it can be easily shown that $d_{s}=2$

\section{Cooperative Alamouti Diversity SCheme With SNR IMBALANCE AND CHANNEL ESTIMATION ERRORS}

In this section, we look at the effect of both SNR imbalance and channel estimation errors on the relay assisted cooperative Alamouti scheme. To evaluate the impact of channel estimation errors, an MMSE estimator is used in the literature, and the channel estimation errors are assumed to be zero-mean complex Gaussian random variables and independent of the channel [11].

From (4) and (5), $\hat{h}_{B}$ and $\hat{h}_{R}$ are random variables with zero mean and variance $\sigma_{B}^{2}=\sigma_{R}^{2}=1+\beta$, and $h_{B}$ and $\hat{h}_{B}$, as well as $h_{R}$ and $\hat{h}_{R}$, are joint complex Gaussian distributed with normalized correlation coefficient $\frac{1}{\sqrt{1+\beta}} \cdot h_{B}$ and $h_{R}$ can then be represented as

$$
h_{B}=\rho \hat{h}_{B}+w_{B}
$$

and

$$
h_{R}=\rho \hat{h}_{R}+w_{R}
$$

where $\rho=\frac{1}{1+\beta} ; w_{B}$ and $w_{R}$ are complex Gaussian random variables with zero means and variance $\sigma_{d}^{2}=\frac{\beta}{1+\beta}$ [12]. The decoded symbol vector can be rewritten as (3).

Note that the previous result of [12] is obtained as a special case when $r=1$. In general it is difficult to find a closedform equation for the probability of error for the cooperative Alamouti diversity scheme with SNR imbalance and channel estimation errors. We show the effect of both by simulation in Section V.

\section{Simulation Results}

Simulation results are provided to show how SNR imbalance and channel estimation errors impact the cooperative OSTBC transmission in i.i.d Rayleigh fading channels.

Fig 1 shows the theoretical and simulated BER versus SNR performance of the cooperative OSTBC (Alamouti) scheme 


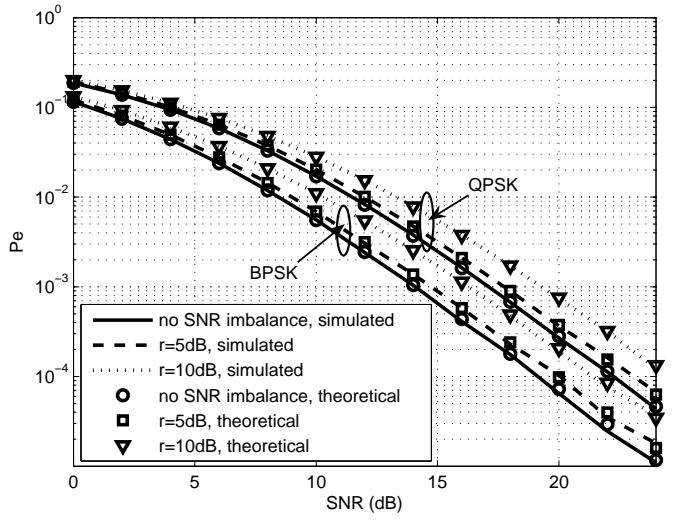

Fig. 1. BER vs. SNR for the cooperative Alamouti scheme for different SNR imbalances $r=0,5 \mathrm{~dB}$ and modulations BPSK and QPSK.

when there is one receive antenna, with different SNR imbalances $r=5,10 \mathrm{~dB}$ and different modulation modes (BPSK and QPSK), for the case of no channel estimation errors. A performance curve for no SNR imbalance $(r=0 \mathrm{~dB})$ case is also shown for reference. By allocating different powers of $\frac{1}{1+r} \gamma$ and $\frac{r}{1+r} \gamma$ to each symbol at the BS and RS respectively, the results in Fig. 1 1 show that the performance degradation is increasing with SNR imbalance. For an SNR imbalance of $10 \mathrm{~dB}$, the performance loss is measured around $2 \mathrm{~dB}$ for a probability of error of $10^{-2}$. More generally, we see from the figure that the curves drawn from the theoretical result (7) perfectly match the simulation curves, showing that the difference between the probabilities of error performance with different SNR imbalance ratio $r$ can be well quantified through our analysis of (7). With regard to the spatial diversity, it is clear in the figure at high SNR region that the cooperative Alamouti scheme achieves a diversity of 2 , which is the same as the regular Alamouti scheme.

Fig. 2 compares the average BER versus SNR for the cooperative Alamouti scheme employing QPSK modulation for different levels of SNR imbalances and Gaussian channel estimation errors. Performance of the cooperative OSTBC system with different system setup ( 2 transmitters at both the BS and RS and 2 receive antennas at the UE) is shown in Fig. 3 It is seen that channel estimation errors drastically affect the performance of the system, especially at high SNR region, showing a more important role than the SNR imbalance. Thus the channel estimation deserves more attention when designing the system.

\section{CONCLUding Remarks}

In this paper, we studied the cooperative OSTBC communication in the second hop of a two-hop decode-and-forward relay assisted cellular network. We investigated the effect of SNR imbalance and channel estimation error on the system performance. A closed-form expression for the probability of error performance is derived to analyze the performance degradation due to the SNR imbalance. It is also proved that

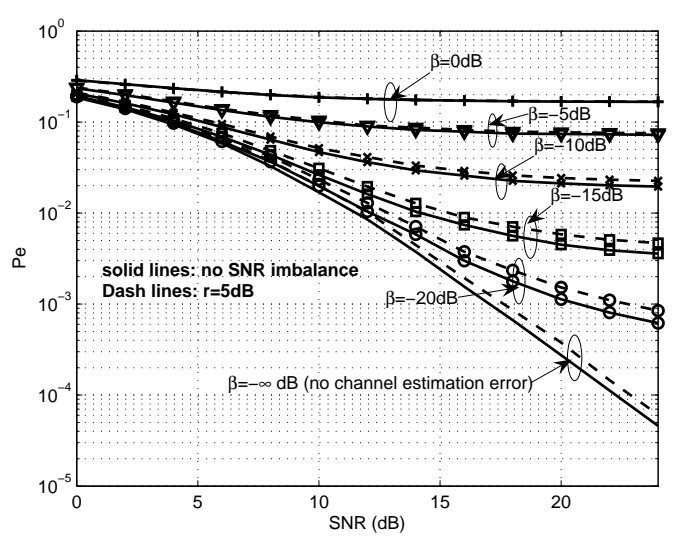

Fig. 2. BER vs. SNR for the cooperative Alamouti scheme for different SNR imbalances $r=0,5 \mathrm{~dB}$ and channel estimation errors, QPSK modulation.

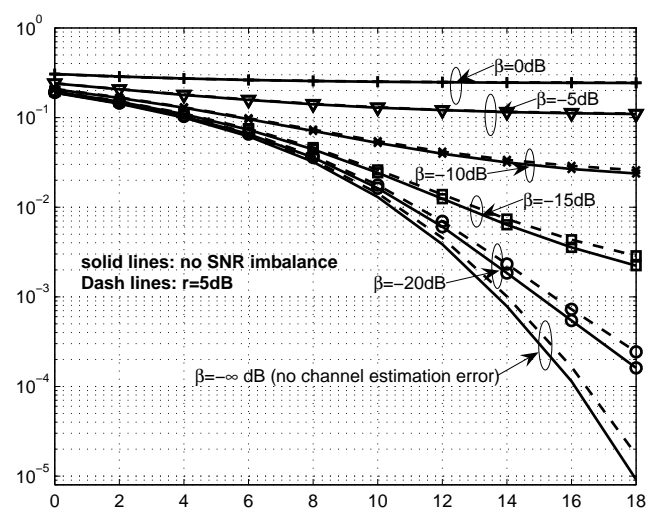

Fig. 3. BER vs. SNR for the cooperative OSTBC scheme $\left(M_{B}=M_{R}=\right.$ $N=2$ ) for different SNR imbalances $r=0,5 \mathrm{~dB}$ and channel estimation errors, 16QAM modulation.

the SNR imbalance, although affecting the system performance, does not change the diversity order. We then show by simulation that channel estimation error has a large effect on the cooperative relay assisted OSTBC communication system and deserves more attention when designing the system.

\section{APPENDIX}

\section{A. Derivation of (7)}

The error probability is given by

$$
P e=E(Q(a \sqrt{\Upsilon}))
$$

where $\Upsilon=\frac{1}{1+r} \gamma\left(\left|h_{B}\right|^{2}+r\left|h_{R}\right|^{2}\right)$ is the instantaneous received SNR and a summation of two independent central Chisquare distributions; $Q(\cdot)$ is Gaussian $\mathrm{Q}$ function defined as

$$
Q(x)=\frac{1}{\pi} \int_{0}^{\frac{\pi}{2}} \exp \left(-\frac{x^{2}}{2 \sin ^{2} \theta} d \theta\right), \quad x \geq 0 .
$$


Thus we have (13) as

$P e=E\left(\frac{1}{\pi} \int_{0}^{\frac{\pi}{2}} \exp \left(-\frac{a^{2} \frac{1}{2} \gamma\left(\frac{2}{1+r}\left|h_{B}\right|^{2}+\frac{2 r}{1+r}\left|h_{R}\right|^{2}\right)}{2 \sin ^{2} \theta} d \theta\right)\right)$.

By changing the order of averaging and integration ( Eq (3.54), [14]) and averaging with respect to $h_{B}$ and $h_{R}$ under the independent Rayleigh fading assumption, we get

$$
\begin{aligned}
P e & =\frac{1}{\pi} \int_{0}^{\frac{\pi}{2}}\left(\frac{1}{1+\frac{a^{2} \frac{1}{2} \gamma \frac{2}{1+r}}{2 \sin ^{2} \theta}}\right)\left(\frac{1}{1+\frac{a^{2} \frac{1}{2} \gamma \frac{2 r}{1+r}}{2 \sin ^{2} \theta}}\right) d \theta \\
& =\frac{1}{\pi} \frac{1}{M N} \frac{1}{\frac{1}{N}-\frac{1}{M}} \int_{0}^{\frac{\pi}{2}}\left(\frac{1}{\frac{1}{\sin ^{2} \theta}+\frac{1}{M}}-\frac{1}{\frac{1}{\sin ^{2} \theta}+\frac{1}{N}}\right) d \theta
\end{aligned}
$$

where $M=\frac{a^{2} \gamma}{2(1+r)}$ and $N=\frac{a^{2} r \gamma}{2(1+r)}$.

It is pointed out in [15] [16] and [17] that

$$
\frac{1}{\pi} \int_{0}^{\frac{\pi}{2}} \frac{1}{\frac{1}{\sin ^{2} \theta}+\frac{1}{M}} d \theta=\frac{1}{2} M\left(1-\frac{1}{\sqrt{1+\frac{1}{M}}}\right),
$$

thus (16) becomes

$$
\begin{aligned}
P e= & \frac{1}{2 M N\left(\frac{1}{N}-\frac{1}{M}\right)}\left(M\left(1-\frac{1}{\sqrt{1+\frac{1}{M}}}\right)\right. \\
& \left.-N\left(1-\frac{1}{\sqrt{1+\frac{1}{N}}}\right)\right)
\end{aligned}
$$

Assume $\mu_{M}=\sqrt{1+\frac{1}{M}}$ and $\mu_{N}=\sqrt{1+\frac{1}{N}}$, we have $\frac{1}{M}=$ $\mu_{M}^{2}-1$ and $\frac{1}{N}=\mu_{N}^{2}-1 ; \mu_{M}=\sqrt{1+\frac{2(1+r)}{a^{2} \gamma}}$ and $\mu_{N}=$ $\sqrt{1+\frac{2(1+r)}{a^{2} \gamma r}}$

The probability of error in 18 is

$$
\begin{aligned}
P e= & \frac{\left(\mu_{M}^{2}-1\right)\left(\mu_{N}^{2}-1\right)}{2\left(\mu_{N}^{2}-\mu_{M}^{2}\right)}\left(\frac{1}{\mu_{M}^{2}-1}\left(1-\frac{1}{\mu_{M}}\right)\right. \\
& \left.-\frac{1}{\mu_{N}^{2}-1}\left(1-\frac{1}{\mu_{N}}\right)\right) \\
= & \frac{1}{2}\left(1-\frac{1}{\mu_{M}}\right)\left(1-\frac{1}{\mu_{N}}\right)\left(1+\frac{1}{\mu_{M}+\mu_{N}}\right) .
\end{aligned}
$$

Plugging $\mu_{M}=\sqrt{1+\frac{2(1+r)}{a^{2} \gamma}}$ and $\mu_{N}=\sqrt{1+\frac{2(1+r)}{a^{2} \gamma r}}$ into (19) completes the proof

\section{REFERENCES}

[1] J. N. Laneman, D. Tse, and G. W. Wornell, "Cooperative diversity in wireless networks: Efficient protocols and outage behavior," IEEE Trans. Inform. Theory, vol. 50, no. 12, pp. 3062-3080, Dec. 2004.

[2] V. Tarokh, H. Jafarkhani, and A. R. Calderbank, "Space-time block codes from orthogonal designs," IEEE Trans. Inform. Theory, vol. 45, pp. 1456-1467, July 1999.

[3] S. M. Alamouti, "A simple transmit diversity technique for wireless communications," IEEE J. Select. Areas Commun., vol. 16, pp. 14511458, Oct. 1998.

[4] J. N. Laneman and G. W. Wornell, "Distributed space-time coded protocols for exploiting cooperative diversity in wireless networks," IEEE Trans. Inform. Theory, vol. 49, no. 10, pp. 2415-2425, Oct. 2003.
[5] R. U. Nabar, H. Bolcskei, and F. W. Kneubuhler, "Fading relay channels: Performance limits and space-time signal design," IEEE J. Select. Areas Commun., vol. 22, pp. 1099-1109, Aug. 2004.

[6] Y. Jing and B. Hassibi, "Distributed space-time coding in wireless relay networks," IEEE Trans. Wireless Commun., vol. 5, no. 12, pp. 35243536, Dec. 2006.

[7] — - "Using orthogonal and quasi-orthogonal designs in wireless relay networks," IEEE Trans. Inform. Theory, vol. 53, no. 11, pp. 4106-4118, Nov. 2007.

[8] G. S. Rajan and B. S. Rajan, "Distributed space-time codes for cooperative networks with partial CSI," IEEE Trans. Inform. Theory, submitted, also available at http://arxiv.org/abs/cs/0701068v1.

[9] L. Zhang and L. J. Cimini., "Power-efficient relay selection in cooperative networks using decentralized distributed space-time block coding," EURASIP J. Advances in Signal Processing, no. 26, Jan. 2008.

[10] InterDigital, "Application of L2 relay in an interference limited environment for LTE-A," 3GPP TSG-RAN WG1_RL1, no. 54, R1-083205, Aug. 2008.

[11] V. Tarokh, A. Naguib, N. Seshadri, and A. R. Calderbank, "Space-time codes for high data rate wireless communications: Performance criteria in the presence of channel estimation errors, mobility and multiple paths," IEEE Trans. Inform. Theory, vol. 47, pp. 199-207, Feb. 1999.

[12] D. Gu and C. Leung, "Performance analysis of transmit diversity scheme with imperfect channel estimation," Electronic Letters, vol. 39, pp. 402403, Feb. 2003.

[13] L. Zheng and D. N. C. Tse, "Diversity and multiplexing: A fundamental tradeoff in multiple antenna channels," IEEE Trans. Inform. Theory, vol. 49, pp. 1073-1096, May 2003.

[14] D. Tse and P. Viswanath, Fundamentals of Wireless Communication. Cambridge University Press, 2005.

[15] Z. Lin, E. Erkip, and A. Stefanov, "Exact pairwise error probability for the MIMO block fading channel," in Proc. Int. Symp. Inform. Theory and its Applications, Parma, Italy, Oct. 2004.

[16] S. Verdu, Multiuser Detection. Cambridge University Press, 1998.

[17] R. Knopp and P. A. Humblet, "On coding for block fading channels," IEEE Trans. Inform. Theory, vol. 46, pp. 189-205, Jan. 2000. 